\title{
REUNI+D: una red universitaria para la construcción colaborativa de conocimiento
}

\section{REUNI+D: a university network for the collaborative construction of knowledge}

\author{
Ana García-Valcárcel Muñoz-Repiso \\ Luis María González Rodero \\ Verónica Basilotta Gómez-Pablos \\ Marta Martín del Pozo \\ Universidad de Salamanca, USAL (España)
}

\section{Resumen}

El movimiento educativo abierto hace referencia al software libre, la formación y los contenidos abiertos o accesibles y la ciencia abierta, considerando la educación como un derecho de todos y un motor de desarrollo social que debe tender a incentivar la construcción y diseminación universal del conocimiento. Con esta finalidad, se construye una Red de Grupos de Investigación consolidados denominada REUNI+D (Red Universitaria de Investigación e Innovación Educativa) (Ref. EDU2015-68718-REDT) que pretende promover, de forma coordinada, acciones de formación abierta y difundir los resultados de investigación e innovación educativa considerando los criterios de la ciencia abierta. En el texto se explican los objetivos, la organización, las acciones desarrolladas, así como el impacto y la valoración de la Red para la construcción conjunta de conocimiento por parte de los miembros implicados. La metodología, de carácter descriptivo, se basa tanto en la revisión documental como en la encuesta de carácter abierto. Los resultados muestran que la valoración de la Red es positiva, tanto para la construcción de conocimiento como para la formación y desarrollo profesional de los investigadores; poniendo de manifiesto que la existencia de REUNI+D ha resultado ser una estrategia clave para reforzar la actividad investigadora de los grupos que la conforman y la difusión de los resultados de la investigación, actuando como una comunidad de práctica y una red de aprendizaje y profesionalización docente.

Palabras clave: conocimiento; red de información; investigación educativa; investigación institucional; educación permanente; universidad abierta.

\begin{abstract}
The open educational movement makes reference to free software, training and open or accessible content and open science, considering education as a fundamental right and a motor of social development which should aim at encouraging the construction and universal dissemination of knowledge. For this purpose, a network of consolidated research groups called REUNI+D (University Network for Research and Educational Innovation) was
\end{abstract}


created (Ref. EDU2014-54943-REDT), which intends to promote, in a coordinated manner, open training courses and disseminate the results of educational research and innovation by considering the criteria of open science. The article explains the objectives, organization, activities, as well as the impact and assessment of the network for the joint construction of knowledge by the involved members. The methodology, of a descriptive character, is based both on the document review and on the survey of open character. The results show that the assessment of the network is positive, both for the construction of knowledge and for the training and professional development of researchers. It shows that the existence of REUNI+D has proven to be a key strategy to reinforce the research activity of the groups that form it and the dissemination of the results of the research, acting as a community of practice and a network of learning and teaching professionalization.

Keywords: knowledge; information network; educational research; institutional research; lifelong learning; open university.

La construcción del conocimiento ha pasado de estar basada en una concepción individual a una consideración de la comunidad como impulsora de la inteligencia distribuida (o cognición distribuida), considerando que los procesos cognitivos pueden ser distribuidos entre los miembros de un grupo social o comunidad (HardyVallée y Payette, 2008; Vuopala, Hyvönen y Järvelä, 2016). Desde esta perspectiva, es necesario que la comunidad universitaria participe de forma activa en el desarrollo de nuevas competencias vinculadas al desarrollo y gestión del conocimiento, potenciando los procesos educativos innovadores y creativos; en este sentido, constituye un factor crítico el diseño e implementación de redes de conocimiento (Sánchez, Pérez y Picco, 2014).

Desde el paradigma de la gestión del conocimiento, que trata de promover el valor del conocimiento real y potencial de los miembros de la comunidad educativa, estableciendo vías de comunicación que posibiliten un buen entendimiento entre los miembros de la comunidad y una posible colaboración con otras instituciones externas, surge la necesidad del trabajo en red (Fernández-Salinero, 2012).

Se trata de una forma de trabajar propia de la Sociedad del Conocimiento, que se basa en la colaboración y la flexibilidad (Velásquez, 2007). Flexibilidad de tiempos, de espacios, de conocimientos, de tareas, de relaciones, etc. Flexibilidad entendida como un valor que el trabajador debe poseer para poder seguir aprendiendo (GámizSánchez y Gallego-Arrufat, 2015; Sevillano y Vázquez-Cano, 2015), una forma de trabajo donde las relaciones con los otros van configurando nuestra percepción de la realidad y de nosotros mismos.

La sociedad digital nos ha traído todas las posibilidades de acceso a la información, formación y comunicación ya mencionadas. Internet ofrece un espacio social compartido en el que cualquier persona puede comunicarse con el resto, un medio universal que facilita la formación permanente, un recurso que supone un gran punto de apoyo para los distintos grupos de investigación universitarios, de cara a 
fomentar el trabajo colaborativo con otras instituciones y organismos universitarios (Khalil y Ebner, 2017; Luna, 2003; Parrilla, 2013; Vera Muñoz, 2014).

En una red todos los participantes pueden enseñar o ser aprendices, todos trabajan de forma conjunta en el logro de los objetivos. El aprendizaje se convierte en un proceso multidireccional, con respecto al acceso a la información y su aplicación en contextos concretos.

Las redes de investigación tienen como objetivo la comunicación y divulgación científica, aprovechando la potencialidad que ofrece internet como recurso comunicativo, y la facilidad para difundir la información y el conocimiento (Arriaga, Minor y Pérez, 2012).

Actualmente existe una gran determinación para la constitución de redes de conocimiento vinculadas a las instituciones de educación universitaria e instituciones de formación permanente; tanto a nivel internacional (Biancani y McFarland, 2013; Gunawardena y otros, 2007; Royero, 2006), como a nivel nacional, donde la constitución de redes de investigación universitarias resulta ser una línea de acción en la mayoría de las universidades (Álvarez, Grau, González y Tortosa, 2016; RoigVila, Mondéjar y Lledó; Sañudo, 2012), tanto en el ámbito de la educación como de la psicología (Lluch, González y Sala, 2017).

Prueba de este interés a nivel estatal son las diferentes convocatorias del Programa Estatal de Fomento de la Investigación Científica y Técnica de Excelencia, vinculadas al Subprograma Estatal de Generación del Conocimiento, desarrolladas en los tres últimos años (Ministerio de Economía, Industria y Competitividad, 2015, 2016 y 2017); resultado de estas convocatorias es la red REUNI+D, objeto del presente artículo.

El trabajo científico realizado en las universidades no queda anclado en sus respectivos archivos, sino que es difundido, fundamentalmente en abierto, para que la sociedad pueda conocer las actividades e investigaciones desarrolladas (López Pérez y Olvera Lobo, 2016), formando parte de ese conocimiento abierto y los procesos de transferencia de información de las universidades a la sociedad.

Si pasamos a analizar las tendencias de futuro, en el último informe Horizon 2017 (Adams y otros, 2017), cabría destacar, en primer lugar, la colaboración para la búsqueda de soluciones efectivas, mediante la creación de redes de conocimiento interuniversitarias, para fomentar el desarrollo del conocimiento de las universidades más allá de los respectivos campus universitarios.

El "movimiento educativo abierto" (Montoya y Aguilar, 2012) que apuesta por los contenidos abiertos se ha ido consolidando, de modo que los materiales con licencias creative common y las prácticas educativas abiertas, como OCW (OpenCourse Ware) o MOOC (Massive Open Online Course), se han multiplicado. En este sentido, los MOOC constituyen una de las tendencias emergentes en la educación superior (Reich, 2015; Vázquez, López y Barroso, 2015; Veletsianos y Shepherdson, 2016) y están atrayendo a una gran variedad de estudiantes de diferentes edades, nacionalidades, intereses, habilidades y contextos (Brahimi y Sarirete, 2015; Johnson, 
2012, 2013). En cualquier caso, lo que interesa destacar es que este movimiento ha sido construido sobre la base de principios en los que se asume que el conocimiento es un bien común (Ehlers, 2011), que pertenece a la humanidad en su conjunto. En ese sentido, se considera a la educación como un motor de desarrollo social que debe tender a incentivar la construcción y diseminación universal del conocimiento.

La Unión Europea se ha interesado en el desarrollo de cursos MOOC, constituyendo una línea prioritaria de la Estrategia 2020 para el fomento del aprendizaje de los ciudadanos basado en estrategias digitales, también del alumnado universitario y profesorado de todos los niveles educativos. Se considera que, a través de estos cursos, se puede plantear un modelo formativo alternativo, con una fuerte orientación de las demandas y estrategias empresariales. En relación con esta iniciativa, se ha creado un portal europeo de MOOC: OpenupEd http://www. openuped.eu) (Vázquez, López y Barroso, 2015).

Chiappe et al. (2015) realizan una revisión sobre las aproximaciones teóricas y prácticas recientes sobre los MOOC. Su trabajo desvela que el conectivismo y el aprendizaje por pares así como la apertura y la relación entre los MOOC y la reutilización de contenido han surgido como tópicos de especial atención desde una perspectiva teórica. En esta línea, se encuentran también los trabajos de Cabero (2013) y Saadatmand y Kumpulainen (2014), quienes defienden que, si bien las posibilidades de la formación virtual para hacer efectiva la formación continua y la actualización profesional son indiscutibles, garantizar la efectividad y calidad de estas modalidades formativas es una cuestión fundamental para las instituciones educativas (García-Sánchez y Gérard-Lojacono, 2016; Ramezan, 2011; Sánchez, Pérez y Picco, 2014).

Por otra parte, en estos momentos las comunidades virtuales son un reto educativo de primer orden y el mundo educativo (investigadores, profesores, alumnos, familias, personas en procesos de formación permanente, agentes sociales...) busca en el espacio virtual nuevas formas de conocimiento de la realidad a través de la interacción con otros. Aunque el término de comunidad evoca una noción espacial relativa a la ubicación geográfica en la cual residen el grupo de personas que la integran, en los escenarios virtuales comprobamos que lo que caracteriza a una comunidad es la cualidad de las relaciones entre sus miembros y los objetivos comunes que comparten (Jerónimo y Aguilar, 2007; Popescu, 2014).

Las comunidades de práctica han sido definidas como redes de personas que comparten un dominio de interés, sobre el cual se comunican online (Cho, 2016; Gannon-Leary y Fontainha, 2007); tienen un carácter profesional, ya que se comparten profesión o condiciones de trabajo. Los miembros se implican activamente en procesos colaborativos de resolución de problemas, apoyándose en la experiencia y en el conocimiento compartido y distribuido entre los miembros de la comunidad. En esta línea surge la Red de investigadores REUNI+D.

Serrat (2010) indica algunos de los valores de las comunidades de práctica como permitir el desarrollo profesional, generar nuevas ideas, resaltar buenas prácticas y 
conectar el aprendizaje a la acción. Ji, Sui y Suo (2017) defienden también que una comunidad de práctica es una eficiente manera para promover la innovación y que, al intercambiar e integrar experiencias, conocimientos e ideas, los miembros de las comunidades de práctica enriquecen y mejoran sus habilidades y experiencias.

También se han señalado los factores para el éxito de estas comunidades, tales como que las actividades que se realicen sean relevantes para los miembros y el dominio, que la gobernanza sea llevada a través de roles y expectativas claras, que la cultura se caracterice por una actitud consistente de compartir y colaborar, que a los miembros se les dé tiempo y se les motive a participar y que se disponga de un medio apropiado de comunicación que añada valor y ayude a llevar a cabo el trabajo (Serrat, 2010).

Si nos centramos en las comunidades de prácticas académicas, como señalan Nistor, Daxecker, Stanciu y Diekamp (2014), la construcción del conocimiento y su compartición no es solamente un medio para alcanzar objetivos prácticos, sino que también se trata propiamente de los objetivos prácticos académicos, tomando relevancia las cuestiones interpersonales y socioemocionales para la aceptación de la compartición del conocimiento. Es decir, se subraya la necesidad de habilidades y conocimiento interpersonal y socioemocional para la construcción de la comunidad.

Existen organizaciones que gestionan algunas redes académicas: el programa de Redes de la UNESCO, UNITWIN (Redes de hermanamiento entre centros); se potencia la investigación, la formación y el desarrollo de programas, a través de la construcción de redes universitarias para fomentar los lazos entre las distintas universidades y también con las enseñanzas medias (Galván-Plata, Almeida y Abdel, 2017; Simmons et al., 2016; Valencia y Trejo, 2016).

El estudio de Patton y Parker (2017) puso de manifiesto que el compromiso en comunidades de práctica provee una base para la colaboración y para reducir el aislamiento, permitiendo a los participantes desarrollar y mejorar sus capacidades de investigación y enseñanza. Además, teniendo en cuenta que el profesorado universitario frecuentemente funciona de manera aislada, lo cual es negativo para el individuo, y para la profesión, las comunidades de práctica se pueden considerar como una herramienta para superar la cultura del aislamiento de la universidad.

Por otrolado, también se hace relevantehablar delas comunidades de aprendizaje, puesto que las comunidades profesionales de aprendizaje de docentes presentan posibilidades como poderosos contextos de desarrollo profesional (Lee, Zhang y Yin, 2011; Popp y Goldman, 2016). Sin embargo, se hace necesaria una cultura de confianza para conseguir el entendimiento y la práctica entre profesores (Lee, Zhang y Yin, 2011). De hecho, los resultados del estudio de Zonoubi, Eslami Rasekh y Tavakoli (2017) demostraron un aumento en la autoeficacia y eficacia colectiva tanto en profesores noveles como experimentados, atribuyendo los profesores este crecimiento a su participación en la comunidad profesional de aprendizaje. 


\section{METODOLOGíA}

El objetivo del trabajo es evaluar el impacto de la Red REUNI+D en relación a la construcción colaborativa del conocimiento, desde una perspectiva objetiva, con base en la producción científica de esta Red y, desde una perspectiva más subjetiva, basándose en la opinión de los participantes en la misma. La hipótesis de la que se parte es que el trabajo en red de los distintos grupos de investigación universitarios, con una orientación hacia el conocimiento abierto, será un buen procedimiento para la construcción de conocimiento y la difusión del mismo a la comunidad educativa.

La metodología, de carácter descriptiva, se basa en la revisión documental y en la encuesta de carácter abierto, aplicada de forma online a los miembros de la Red, especialmente dirigida a los coordinadores de los grupos de investigación. La muestra encuestada es de 14 sujetos (personal docente e investigador) que representan a los 10 grupos de investigación educativa que conforman la Red REUNI+D (Barcelona, Complutense, Extremadura, Granada, La Laguna, Málaga, País Vasco, Salamanca y Santiago de Compostela).

La encuesta se configura con tres preguntas abiertas de carácter indagatorio:

1. ¿Qué aporta REUNI+D a la construcción conjunta de conocimiento?

2. ¿Qué te aporta personalmente REUNI+D en tu formación y desarrollo profesional?

3. ¿Consideras que REUNI+D es una buena estrategia para crear conocimiento? ¿por qué?

Para llevar a cabo el análisis cualitativo de las respuestas, utilizamos el análisis de contenido, una técnica para interpretar el contenido de los textos. Para ello, se ha elaborado un sistema de categorías, el cual ha sido validado por dos expertos, y se ha sometido a control a través de la doble categorización por parte de dos investigadores, obteniendo un alto índice de fiabilidad. Para el análisis del contenido se ha utilizado el programa NVivo11, que ha permitido obtener las frecuencias de las categorías, así como un árbol de palabras y frases.

En cuanto a la revisión documental, se han analizado todos los documentos disponibles en la web de la REUNI+D hhttp://reunid.eu/la-red/4 y en los informes de investigación presentados al Ministerio como parte del proyecto REUNI+D.

\section{RESULTADOS}

\section{Impacto y construcción del conocimiento a través de REUNI+D}

Una vez que la Red se ha consolidado y lleva trabajando en diversos proyectos a lo largo de diez años, su contribución a la construcción de conocimiento en el 
campo de la innovación educativa es significativa. Prueba de ello son las actividades de formación, tanto de carácter general como las orientadas a la realización de tesis doctorales, proyectos de investigación en marcha, publicaciones, etc. Estas actividades de formación pueden consultarse en la web de la Red ya mencionada.

La construcción del conocimiento en el ámbito de la educación se focaliza en los siguientes objetivos de REUNI+D: 1) Contribuir a la formación de jóvenes investigadores a través de cursos, seminarios, escuelas de verano, webinars, MOOC, etc.; 2) Fomentar el desarrollo de métodos de investigación innovadores que den cuenta de los complejos problemas y fenómenos del ámbito de la educación; 3) Promover iniciativas que permitan la transferencia del conocimiento a todos los agentes interesados en la educación (responsables políticos, formadores, asesores, docentes, familias, estudiantes,...) y 4) Promover iniciativas creativas de construcción del conocimiento que contribuyan a afrontar los retos de la educación de forma colectiva.

Las acciones que se desenvuelven en el contexto de la Red para contribuir al avance del conocimiento de una forma colaborativa y abierta son las siguientes: 1) participación en proyectos conjuntos de I+D+I, tanto en las convocatorias nacionales, como en las europeas o internacionales; 2) organización de actividades formativas y de difusión, tales como encuentros nacionales e internacionales que contribuyan al intercambio de resultados de investigación y el avance del conocimiento; 3) Compartir y validar metodologías e instrumentos de investigación educativa que respondan a los objetivos de la Red y de los grupos de investigación que la componen (Correa, Fernández, Gutiérrez-Cabello, Losada y Ochoa-Aizpurua, 2015).

A través de todas estas acciones, REUNI+D contribuye a desarrollar y potenciar nuevas líneas de investigación y nuevas perspectivas de análisis de la realidad educativa. El trabajo realizado ha contribuido a desarrollar nuevas líneas y métodos de investigación y, en consonancia, nuevos análisis de la realidad educativa, en colaboración con entidades como el ESRC National Centre for Research Methods de Gran Bretaña o The Centre for the Learnig Sciences de Alemania.

Sehan generado proyectos einiciativas nacionales einternacionales deproducción y transferencia del conocimiento mucho más potentes que los desarrollados de forma específica por cada uno de los grupos de investigación.

La propuesta de las actuaciones, generadas desde los proyectos de investigación y las iniciativas de transferencia de conocimiento que se llevan a cabo de forma conjunta, tienen una mayor repercusión en el contexto educativo a través de la difusión en diferentes foros, tanto nacionales como internacionales. Esto está contribuyendo a:

- Una mayor visibilidad e internacionalización de los resultados de la investigación educativa, al agrupar la producción científica, facilitando el acceso y creando sinergias que redundan en un mayor impacto de la misma. 
- El fortalecimiento de las estructuras investigadoras al facilitar el intercambio de conocimientos, la colaboración en actividades de investigación y su difusión y el uso compartido de recursos humanos y tecnológicos.

- El fomento de la competitividad de los grupos de investigación, al dotarles de una mejor estructura para participar en las convocatorias de proyectos nacionales e internacionales y en los foros propios de la comunidad científica.

- El fomento de la colaboración con otras redes e instituciones del ámbito internacional.

- La creación de espacios y recursos virtuales y presenciales de formación e intercambios para los investigadores, tanto noveles como expertos.

\section{Valoración de REUNI+D por los miembros de la Red}

Las respuestas a las tres cuestiones planteadas en la encuesta, emitidas mayoritariamente por los directores de los grupos de investigación en representación de los miembros de la Red, apuntan a una valoración positiva de la Red, tanto para la construcción conjunta de conocimiento como para la formación y desarrollo profesional de los investigadores, poniendo de manifiesto que la existencia de REUNI+D ha resultado ser una buena estrategia para crear conocimiento.

Exploramos, en primer lugar, las palabras que aparecen con más frecuencia en el discurso de los participantes con el programa Nvivo11 y encontramos que las más destacadas son: investigación (33 referencias), red (16 referencias), diferentes (15 referencias), conocimiento (14 referencias), educación (14 referencias), grupos (14 referencias) y proyectos (13 referencias). La frecuencia de estas palabras anticipa la relación encontrada entre pertenecer a la Red, potenciar la investigación, ampliar los puntos de vista entre diferentes y construir el conocimiento.

Posteriormente, escogemos la palabra clave "investigación", como palabra más citada para analizar en su contexto, obteniendo el árbol de palabras y frases que se muestra en la figura 1. Este árbol contiene las frases de los participantes en las que aparece el término seleccionado. 
Figura 1. Árbol de palabras y frases de la palabra clave "investigación”

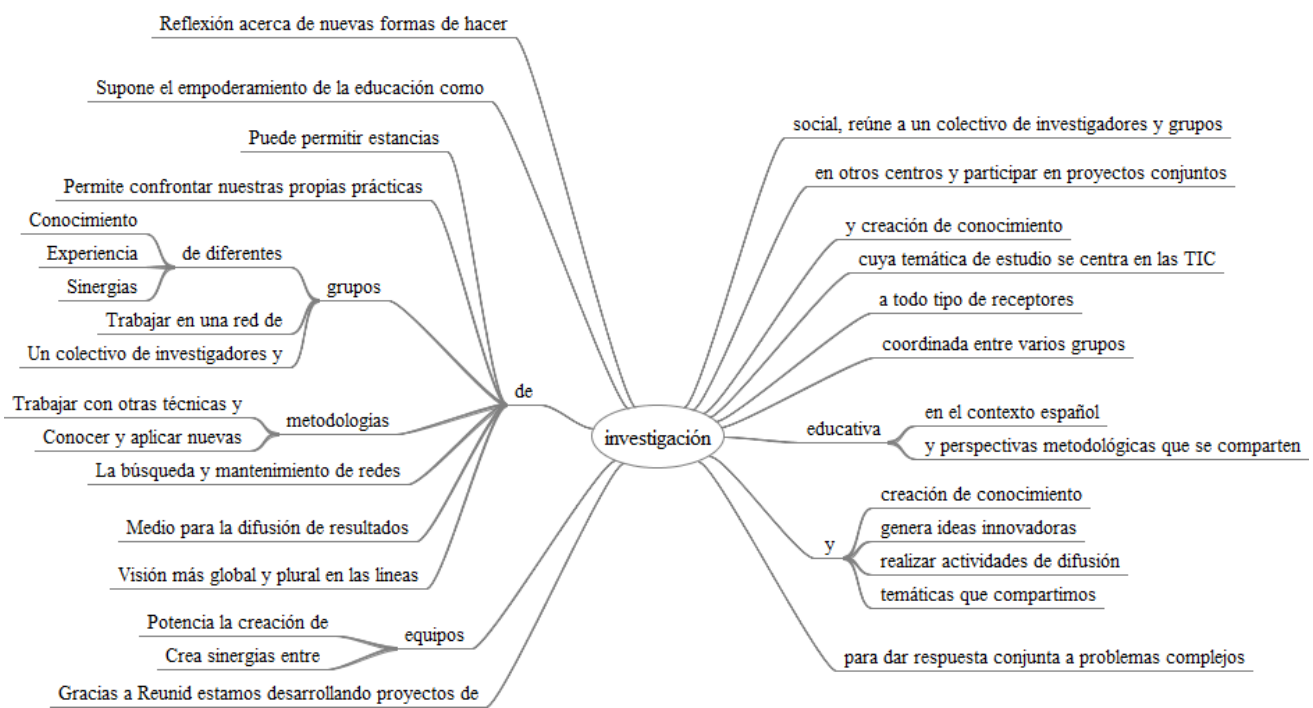

Se pueden resaltar del árbol algunas ideas que nos parecen de especial interés en el análisis del tema y que resumen las opiniones expresadas por los docentes e investigadores de nuestro estudio, en relación al potencial de REUNI+D como red de investigación: "Reflexión acerca de nuevas formas de hacer investigación”; "Supone el empoderamiento de la educación como investigación"; "Permite confrontar nuestras propias prácticas de investigación”; "Trabajar con otras técnicas y metodologías de investigación"; "La búsqueda y mantenimiento de redes de investigación”; "Medio para la difusión de resultados de investigación"; "Visión más global y plural en las líneas de investigación"; "Investigación educativa en el contexto español", "Investigación y creación de conocimiento"; "Investigación para dar respuesta a problemas complejos”.

Se realiza una codificación de todas las respuestas, identificando 7 supracategorías y 29 categorías de forma inductiva. A continuación, obtenemos el número y porcentaje de referencias textuales de cada una de las categorías identificadas. Tal y como podemos observar en la tabla 1, las categorías que los docentes e investigadores mencionan con más frecuencia son el trabajo colaborativo (20 referencias), proyectos de investigación (12 referencias) y diversidad de puntos de vista (8 referencias). 
Tabla 1. Valoración de REUNI+D como estrategia para la creación de conocimiento (sistema de categorías, frecuencias y porcentajes)

\begin{tabular}{|c|c|c|}
\hline Sistema de categorias & $\begin{array}{c}\text { Referencias } \\
\text { textuales }\end{array}$ & $\%$ \\
\hline 1. Espacio para la colaboración & 37 & $\mathbf{2 9 , 3 6}$ \\
\hline 1.1. Conocimiento de compañeros & 4 & 5,04 \\
\hline 1.2. Crea una comunidad de práctica & 2 & 2,52 \\
\hline 1.3.Facilita la comunicación & 4 & 5,04 \\
\hline 1.4. Reflexión conjunta & 5 & 6,03 \\
\hline 1.5. Resolución de problemas & 2 & 2,52 \\
\hline 1.6.Trabajo en colaboración & 20 & 25,2 \\
\hline 2. Apertura de la mirada & 20 & $\mathbf{1 5}, \mathbf{8 7}$ \\
\hline 2.1.Diversidad de puntos de vista & 8 & 10,06 \\
\hline 2.2. Multidisciplinariedad & 2 & 2,52 \\
\hline 2.3. Perspectiva innovadora y creativa & 3 & 3,78 \\
\hline 2.4. Visión global y plural & 5 & 6.03 \\
\hline 3. Conocimiento a través de acciones formativas & 16 & 12,69 \\
\hline 3.1.Aprendizaje compartido & 4 & 5,04 \\
\hline 3.2. Cursos conjuntos & 3 & 3,78 \\
\hline 3.3. Formación científica & 4 & 5,04 \\
\hline 3.4. Metodologías docentes & 2 & 2,52 \\
\hline 3.5. Transferencia aprendizajes & 2 & 2,52 \\
\hline 4. Conocimiento a través de investigaciones & 23 & $\mathbf{1 8 , 2 5}$ \\
\hline 4.1. Estímulo para avanzar & 4 & 5,04 \\
\hline 4.2. Metodologías de investigación & 2 & 2,52 \\
\hline 4.3. Producción de conocimiento & 4 & 5,04 \\
\hline 4.4. Proyectos de investigación & 12 & 15,12 \\
\hline 5. Recursos universitarios & $\mathbf{1 1}$ & $\mathbf{8 , 7 3}$ \\
\hline 5.1. Apoyo económico & 2 & 2,52 \\
\hline 5.2. Contactos de trabajo & 2 & 2,52 \\
\hline 5.3. Creación de equipos de investigación & 3 & 3,78 \\
\hline 5.4. Espacios de encuentro & 3 & 3,78 \\
\hline 6. Red de profesionales & 10 & 7,93 \\
\hline 6.1. Empoderamiento colectivo & 3 & 3,78 \\
\hline 6.2. Equipos interuniversitarios & 2 & 2,52 \\
\hline 6.3. Experiencia de diferentes grupos & 3 & 3,78 \\
\hline 6.4. Expertos en la temática & 1 & 1,26 \\
\hline
\end{tabular}




\begin{tabular}{|l|c|c|}
\hline \multicolumn{1}{|c|}{ Sistema de categorias } & $\begin{array}{c}\text { Referencias } \\
\text { textuales }\end{array}$ & $\%$ \\
\hline 7. Difusión de resultados & $\mathbf{9}$ & $\mathbf{7 , 1 4}$ \\
\hline 7.1.Publicaciones conjuntas & 5 & 6,03 \\
\hline 7.2. Visibilización como red & 4 & 5,04 \\
\hline Total & $\mathbf{1 2 6}$ & $\mathbf{1 0 0}$ \\
\hline
\end{tabular}

Los participantes de este estudio consideran que REUNI+D brinda un contexto ideal que fomenta la colaboración, la formación de relaciones y la interactividad entre diferentes profesionales de la educación: "Un espacio para la colaboración y el trabajo conjunto, con una gran riqueza"; "Colaboraciones expandidas, conexión con colegas y aprendizaje de otras formas de hacer"; "Hoy en día no se puede crear nada de forma aislada, necesitamos encontrarnos para contaminarnos y en ese camino juntos, comenzar a producir, el trabajo compartido siempre es enriquecedor"; "Colaborar, participar y unir esfuerzos, proyectos, subvenciones y difusión científica siempre suma"; "Trabajo cooperativo entre distintos profesionales para poder entender y estudiar los aspectos relevantes del mundo educativo desde distintas perspectivas y enfoques".

En segundo lugar, perciben que REUNI+D fomenta la creación de proyectos de investigación como una oportunidad para producir conocimiento, profundizar en ideas y teorías educativas relevantes, así como conocer nuevas metodologías y fomentar su difusión: "Gracias a REUNI+D estamos desarrollando proyectos de investigación coordinados entre varios grupos, que potencia el trabajo"; "Desarrollar proyectos de investigación con diferentes grupos de la red, así como colaborar en publicaciones conjuntas de nuestro grupo"; "Participar en proyectos conjuntos con otras universidades, lo que contribuye a mi formación y desarrollo personal".

Por último, opinan que esta red les permite conocer a otros profesionales de la educación con diferentes enfoques y puntos de vista acerca de la docencia e investigación, que enriquece su trabajo día a día: "Conocer las diferentes visiones de la investigación educativa y las nuevas perspectivas metodológicas, así como conocer cómo trabajan otros, abre la mirada"; "Oportunidad de disfrutar de la diversidad de puntos de vista de personas valiosas e innovadoras"; "Es posible ver la educación y las prácticas investigadoras desde puntos de vista muy diferentes".

\section{DISCUSIÓN Y CONCLUSIONES}

En una sociedad Red, en términos de Castells (1997, 2005), el conocimiento se genera y difunde cada vez más en estructuras en red de carácter horizontal y con nodos distribuidos en el espacio que contribuyen a un objetivo común. La organización en red se caracteriza por la flexibilidad y "por una cultura de la virtualidad real construida mediante un sistema de medios de comunicación omnipresentes, interconectados 
y diversificados, y por la transformación de los cimientos materiales de la vida, el espacio y el tiempo" (Castells, 1997, p. 23).

Las universidades, como instituciones y lugares de referencia de la actividad docente e investigadora, deben preocuparse por el proceso de gestión del conocimiento con el objeto de mejorar la capacidad para acceder, analizar y usar la información por parte de la comunidad educativa, así como de reestructurar las actividades para mejorar los procesos de investigación y de enseñanza-aprendizaje. Una idea clave, en los modelos de gestión del conocimiento, es que los trabajadores deben aportar conocimientos al proceso de producción, además de participar en el análisis y solución de los problemas. En el ámbito educativo, a los profesores se les exigiría un aporte intelectual, creatividad, investigación, innovación, asumir responsabilidades, generar competencias sociales, difundir el conocimiento, etc.

En el caso que se ha referido, REUNI+D se ha constituido como una Red de grupos de investigación en el campo de la educación y la innovación educativa para fomentar el trabajo colaborativo entre investigadores de diversas universidades, y aprovechar las sinergias de la actividad formativa e investigadora para obtener una mayor proyección nacional e internacional, así como poner en marcha una nueva forma de construir conocimiento, en la línea de la denominada Ciencia Abierta.

La Ciencia Abierta (Open Science) representa un enfoque para investigar que es colaborativo, transparente y accesible (ver Open Science "Wheel"). Se caracteriza por ser Disponible, Accesible, Interoperable y Reutilizable. Nosek et al. (2015) señalan ocho estándares como guía de promoción de la transparencia y la apertura de la ciencia: estándares relativos a la citación, la transparencia de los datos, la transparencia en los materiales de investigación, la transparencia en el diseño y análisis, el registro previo de los estudios, el registro previo de los planes relativos al análisis de los datos, la transparencia en los métodos de análisis y la realización de estudios de replicación. En este sentido, considerar estos estándares contribuye al desarrollo de la ciencia, pero también, como señala McKiernan et al. (2016), la investigación y la ciencia abierta pueden contribuir al desarrollo profesional de los propios investigadores a través del incremento en las citas, atención de los medios, nuevas oportunidades de trabajo y de financiación. Por ello, la Red se plantea desarrollar entre sus investigadores competencias relacionadas con la Ciencia Abierta (O'Carroll et al., 2017).

REUNI+D también se puede valorar como una red de aprendizaje, entendida como un entorno de aprendizaje en línea que ayuda a los participantes a desarrollar sus competencias colaborando y compartiendo información (Sloep y Berlanga, 2011). Como señalan estos autores, las redes de aprendizaje están diseñadas para tratar de enriquecer la experiencia de aprendizaje en los contextos profesionales. En su empeño para adquirir competencias, los usuarios de una red de aprendizaje pueden (Koper, 2009): Intercambiar experiencias y conocimiento con otros; Trabajar en colaboración en proyectos (p. ej., de innovación, investigación, trabajos); Crear grupos de trabajo, comunidades, debates y congresos; Ofrecer y recibir apoyo a/de 
otros usuarios de la red de aprendizaje (como dudas, observaciones, etc.); Evaluarse a sí mismos y a otros, buscar recursos de aprendizaje, crear y elaborar sus perfiles de competencias.

En la línea que señalan Jara (2012) y Velandia-Mesa, Serrano-Pastor y Martínez-Segura (2017), la articulación entre la investigación educativa y la práctica profesional requiere llevar a cabo la sistematización de la experiencia pedagógica, entendida como un ejercicio permanente de producción de conocimiento crítico desde la práctica.

El trabajo en red que se plantea REUNI+D, como conjunto de diez grupos de investigación educativa y su futura conexión con otras redes, pretende empoderar la investigación educativa como elemento básico en la innovación de la práctica educativa tanto en nuestro país como en contextos internacionales, principalmente europeos y latinoamericanos. El rápido cambio social y tecnológico que caracteriza nuestra sociedad hace indispensable el desarrollo de investigaciones que analicen de forma rigurosa el despliegue de las tecnologías en los diferentes contextos educativos y su repercusión en la formación, conocimiento, comportamientos, seguridad, etc. de los ciudadanos. La transformación digital de las instituciones educativas tiene que ser facilitada y acompañada por políticas públicas, entre ellas las orientadas a fomentar la investigación educativa y la difusión de los resultados. En este objetivo, se asienta la necesidad de potenciar las redes de investigación educativa.

\section{Agradecimiento}

El trabajo se enmarca en un proyecto de investigación de la convocatoria del Programa Estatal de Fomento de la Investigación Científica y Técnica de Excelencia, Subprograma Estatal de Generación de Conocimiento, Modalidad Acciones de dinamización "Redes de Excelencia". REUNI+D (Red Universitaria de Investigación e Innovación Educativa) (Ref. EDU2014-54943-REDT). Agradecemos a los directores y miembros de los grupos de investigación su colaboración en el trabajo.

\section{REFERENCIAS BIBLIOGRÁFICAS}

Adams Becker, S., Cummins, M., Davis, A., Freeman, A., Hall Giesinger, C., y Ananthanarayanan, V. (2017). NMC Horizon Report: 2017 Higher Education Edition. Austin, Texas: The New Media Consortium. Recuperado de https:/ www.nmc.org/publication/nmc-horizonreport-2017-higher-education-edition/

Álvarez, J. D., Grau, S., González, C., y Tortosa, M. T. (2016). El Programa Redes de Investigación en Docencia Universitaria
(REDES): evolución y evaluación. Atas Investigação Qualitativa em Educação, 379-388. Porto, Portugal: Universidad de Porto. ( $5^{\circ}$ Congresso Ibero-Americano em Investigação Qualitativa (CIAIQ2016), 12 a 14 de julio de 2016). Recuperado de http://proceedings.ciaiq.org/index.php/ ciaiq2016/article/view/622

Arriaga Méndez, J., Minor Jiménez, M. G. y Pérez Cervantes, M. L. (2012). Retos y desafíos de las redes de investigación. 
Revista Iberoamericana sobre Calidad, Eficacia y Cambio en Educación, 10(3), 178-183.

Biancani, S., y McFarland, D. A. (2013). Social Networks Research in Higher Education. In M. B. Paulsen (Ed.), Higher Education: Handbook of Theory and Research (151215). Dordrecht: Springer. doi: https:// doi.org/10.1007/978-94-007-5836-0

Brahimi, T., y Sarirete, A. (2015). Learning outside the classroom through MOOCs. Computers in Human Behavior, 51, 604609. doi: 10.1016/j.chb.2015.03.013

Castells, M. (1997). La era de la información. Economía, sociedad y cultura. Madrid: Alianza Editorial.

Castells, M. (2005). LaEradela Información. Vol. I: La Sociedad Red. Madrid: Alianza Editorial.

Cho, H. (2016). Under co-construction: An online community of practice for bilingual pre-service teachers. Computers and Education, 92-93, 76-89.

DePablos-Pons, J., Colás-Bravo, P., GonzálezRamírez, T., y Conde-Jiménez, J. (2015). Dimensiones en las que fundamentar la formación investigadora en Tecnología Educativa. RELATEC, 14(1), 57-74. doi: http://dx.doi.org/10.17398/1695 288X.14.1.57

Cabero, J. (2013). La formación virtual en el Nuevo entramado 2.0: el e-learning 2.o. En I. Aguaded y J. Cabero (Coord.), Tecnología y medios para la educación en la e-sociedad. Madrid: Alianza Editorial, (23-51).

Chiappe, A., Hine, N., y Martínez, J. A. (2015). Literatura y práctica: una revisión crítica acerca de los MOOC. Comunicar, 44, 9-18. doi: http://dx.doi.org/10.3916 C44-2015-01

Correa, J. M., Fernández, L., GutiérrezCabello, A., Losada, D., y Ochoa-Aizpurua, B. (2015) Formación del Profesorado, Tecnología Educativa e Identidad Docente Digital. RELATEC, 14(1), 45-56. doi: http://dx.doi.org/10.17398/1695 288X.14.1.45

Ehlers, U. D. (2011). Extending the Territory: From Open Educational Resources to Open Educational Practices. Journal of Open, Flexible and Distance Learning, 15(2), 1-10.

Fernández-Salinero, C. (2012) Cómo gestionar el nuevo conocimiento pedagógico. En L. García Aretio (Ed.), Sociedad del conocimiento y Educación . Madrid: UNED, (61-68).

Galván-Plata, M. E., Almeida Gutiérrez, E., y Abdel Salamanca-Gómez, F. (2017). Formación de redes institucionales de investigación como estrategia de mejora. Revista Médica del Instituto Mexicano del Seguro Social (IMSS), 55(3), 374-378.

Gámiz-Sánchez, V., y Gallego-Arrufat, M. J. (2016). Modelodeanálisis de metodologías didácticas semipresenciales en Educación Superior. Educación XX1, 19(1), 39-61. doi: 10.5944/ educXX1.13946.

Gannon-Leary, P., y Fontinha, E. (2007). Communities of Practice and virtual learning communities: benefits, barriers and success factors, eLearning Papers, 5. Recuperado de http://elearningpapers. eu/en/download/file/fid/21095

García-Sánchez, S., y Gérard-Lojacono, F. (2016). Aprendiendo fuera del aula. Los MOOC y el conocimiento Ubicuo. En P. Gómez Hernández, A. García Barrera y C. Monge López (Eds.), La cultura de los MOOC. Madrid: Síntesis, (99-115).

Gunawardena, C. N., Hermans, M. B., Sanchez, D., Richmond, C., Bohley, M., y Tuttle, R. (2009). A theoretical framework for building online communities of practice with social networking tools. Educational Media International, 46(1), 3-16.

Hardy-Vallée, B., y Payette, N. (2008). Beyond the brain: embodied, situated, and distributed cognition. Cambridge: Scholars Pub. 
Jara, O. (2012). Sistematización de experiencias, investigación y evaluación: aproximaciones desde tres ángulos. The International Journal for Global and Development Education Research, 1, 5670.

Jerónimo, J. A. y Aguilar, E. (2007). En busca de la comunidad de aprendizaje en Red, recuperando la práctica en la interacción virtual. Congreso Virtual Educa 2007, Brasil.

Ji, H., Sui, Y. T., y Suo, L. L. (2017). Understanding innovation mechanism through the lens of communities of practice (COP). Technological Forecasting \& Social Change, 118, 205212. doi: 10.1016/j.techfore.2017.02.021.

Johnson, L., Adams, S., y Cummins, M. (2012). The NMC Horizon Report: 2012 Higher Education Edition. Austin, Texas: The New Media Consortium. Recuperado de https://www.nmc.org/publication/ nmc-horizon-report-2012-higher-ededition/

Johnson, L., Adams Becker, S., Cummins, M., Estrada, V., Freeman, A., y Ludgate, H. (2013). NMC Horizon Report: 2013 Higher Education Edition. Austin, Texas: The New Media Consortium. Recuperado de https://www.nmc.org/publication/ nmc-horizon-report-2013-highereducation-edition/

Khalil, H., y Ebner, M. (2017). Using Electronic Communication Tools in Online Group Activities to Develop Collaborative Learning Skills. Universal Journal of Educational Research, 5(4), 529-536. doi: 10.13189/ujer.2017.050401.

Koper, R. (Ed.). (2009). Learning Network Services for Professional Development. Berlin: Heidelberg: Springer.

Lee, J. C. K., Zhang, Z., y Yin, H. (2011). A multilevel analysis of the impact of a professional learning community, faculty trust in colleagues and collective efficacy on teacher commitment to students.
Teaching and Teacher Education, 27, 820-830. doi: 10.1016/j.tate.2011.01.006. López Pérez, L., y Olvera Lobo, M. D. (2016). Comunicación pública de la ciencia a través de la web 2.o. El caso de los centros de investigación y universidades públicas de España. Revista El Profesional de la Información, 25(3), 441-448. doi: https://doi.org/10.3145/epi.2016.may.14

Lluch, O., González, J., y Sala, F. (2017). Evolución de las redes científicas y grupos de investigación. El caso de la psicología educativa en España durante los quinquenios 2004-2008 y 20092013. Revista Anales de Psicología, 33(2), 356-364. Recuperado de http:/ revistas.um.es/analesps/article/view/ analesps.33.2.249891

Luna, M. (Coord.)(2003). Itinerarios de conocimiento, formas dinámicas $y$ contenido: un enfoque de redes. Barcelona: Arthropos.

Mackey, J., y Evans, T. (2011). Interconnecting Networks of Practice for Professional Learning. International Review of Research in Open and Distance Learning, 12(3), 1-18. doi: 10.19173/ irrodl.v12i3.873.

McKiernan, E. C., Bourne, P. E., Brown, C. T., Buck, S., Kenall, A., Lin, J., McDougall, D., Nosek, B. A., Ram, K., Soderberg, C. K., Spies, J. R., Thaney, K., Updegrove, A., Woo, K. H., y Yarkoni, T. (2016). How open science helps researchers succeed. eLife, 5, e16800. doi: 10.7554/ eLife.1680o.

Ministerio de Economía, Industria y Competitividad (2015). Acciones de dinamización "Redes de Excelencia" 2015. Programa Estatal de Fomento de la Investigación Científica y Técnica de Excelencia. Subprograma Estatal de Generación de Conocimiento. Recuperado de https://goo.gl/L7U4ko

Ministerio de Economía, Industria y Competitividad (2016). Acciones de dinamización "Redes de Excelencia" 
2016. Programa Estatal de Fomento de la Investigación Científica y Técnica de Excelencia. Subprograma Estatal de Generación de Conocimiento. Recuperado de https://goo.gl/qTH5fJ

Ministerio de Economía, Industria y Competitividad (2017). Acciones de dinamización "Redes de Excelencia" 2017. Programa Estatal de Fomento de la Investigación Científica y Técnica de Excelencia. Subprograma Estatal de Generación de Conocimiento. Recuperado de https://goo.gl/s7baS3

Montoya, M. S., y Aguilar, J. V. (2012). Movimiento Educativo Abierto. Recuperado de http://goo.gl/4F6KWA

Nistor, N., Daxecker, I., Stanciu, D., y Diekamp, O. (2014). Sense of community in academic communities of practice: predictors and effects. Higher Education, 69(2), 257-273. doi: 10.1007/s10734-0149773-6.

Nosek, B. A., Alter, G., Banks, G. C., Borsboom, D., Bowman, S. D., Breckler, S. J., y Yarkoni, T. (2015). Promoting an open research culture, Science, 348(6242), 1422-1425. doi: 10.1126/science.aab2374.

O'Carroll, C., Kamerlin, C. L., Brennan, N., Hyllseth, B., Kohl, U., O’Neill, G., y Van Den Berg, R. (2017). Providing researchers with the skills and competencies they need to practice Open Science. Luxembourg: European Commission. Publications Office of the European Union. Recuperado de http:// ec.europa.eu/research/openscience/ pdf/os skills wgreport final. pdf\#view=fitypagemode=none

Parrilla, M. A. (2013). Equidad e innovación en la investigación educativa: reflexiones y aportaciones desde la red de investigación CIES. Revista de Investigación en Educación, 11(3), 7-13.

Patton, K., y Parker, M. (2017), Teacher education communities of practice: More than a culture of collaboration. Teaching and Teacher Education, 67, 351-36o. doi: 10.1016/j.tate.2017.06.013.

Popescu, E. (2014). Providing collaborative learning support with social media in an integrated environment. World Wide $W e b, 17(2), 199-212$. doi: https://doi. org/10.1007/s11280-012-0172-6

Popp, J. S., y Goldman, S. R. (2016). Knowledge building in teacher professional learning communities: Focus of meeting matters, Teaching and Teacher Education, 59, 347-359. doi: 10.1016/j.tate.2016.06.007.

Ramezan, M. (2011). Intellectual capital and organizational organic structure in knowledge society: how are these concepts related? International Journal of Information Management, 31, 88-95.

Reich, J. (2015). Rebooting MOOC Research. Science, 347(6217), 34-35. doi: 10.1126/ science.1261627.

REUNI+D (2017). Red Universitaria de Investigación e Innovación Educativa. Cambios Sociales y Retos para la Educación en la Era Digital. Recuperado de http://reunid.eu

Roig-Vila, R., Mondéjar, L., y Lledó, G. L. (2016). Redes sociales científicas. La Web social al servicio de la investigación. IJERI: International Journal of Educational Research and Innovation, 5, 170-183. Recuperado de https:/ www.upo.es/revistas/index.php/IJERI/ article/view/1615

Royero, J. D. (2006). Las redes de Investigación y desarrollo (I+D) como estrategia de uso de las TIC en las universidades de América Latina. Revista de Universidad y Sociedad del Conocimiento (RUSC), 3(2), 1-15. doi: http://dx.doi.org/10.7238/rusc.v3i2.280

Saadatmand, M., y Kumpulainen, K. (2014). Participants' Perceptions of Learning and Networking in Connectivist MOOC. MERLOT. Journal of Online Learning and Teaching, 1O(1), 16-30. 
Sánchez Ambriz, G., Pérez Balbuena, J. d. J., y Picco Troncoso, L. L. (2014). Redes de conocimiento basadas en la gestión de conocimiento: creación y organización para la docencia e investigación universitaria. Revista Interamericana de Bibliotecnología, 37(3), 215-225.

Sañudo, L. (2012). El papel de las redes profesionales de investigación en un mundo globalizado. Revista Electrónica Iberoamericana sobre Calidad, Eficacia y Cambio en Educación (REICE), 10(3), 135-143. Recuperado de https://dialnet. unirioja.es/descarga/articulo/4121095. pdf

Serrat, O. (2010). Building communities of practice. Washington, DC: Asian Development Bank.

Sevillano, M. L., y Vázquez-Cano, E. (2015). Modelos de investigación en contextos ubicuos y móviles en educación superior. Education Siglo XXI, 33(2), 329-332.

Simmons, J. A., Anderson, L. J., Bowne, D. R., Dosch, J. J., Gartner, T. B., Hoopes, M. F., Kuers, K., Lindquist, E., Mccay, T. S., Pohlad, B., Thomas, C. L., y Shea, K. (2016). Collaborative Research Networks Provide Unique Opportunities for Faculty and Student Researchers. Council on Undergraduate Research Quarterly, 36(4), 12-18.

Sloep, P., y Berlanga, A. (2011). Redes de aprendizaje, aprendizaje en red. Comunicar, 37, 55-64. doi: https://doi. org/10.3916/C37-2011-02-05

Sun, Z., Liu, R., Luo, L., Wu, M., y Shit, C. (2017). Exploring collaborative learning effect in blended learning environments. Journal of Computer Assisted Learning, 33(6), 575-587.

Valencia Valencia, A., y Trejo Cázares, M. d. C. (2016). Academic and research networks management: challenges for higher education institutions in Mexico. International Journal of Educational
Technoloqu in Hiqher Education, 13, 1-11. doi: https://doi.org/10.1186/s41239-0160013-2

Vázquez Cano, López Meneses, E., y Barroso Osuna, J. (2015). El futuro de los MOOC. Retos de la formación on-line, masiva y abierta. Madrid: Síntesis.

Velásquez, L. A. (2007). Las redes de investigación virtuales: propuesta de fomento y desarrollo de la cultura investigativa en las instituciones de educación superior. Revista de Universidad y Sociedad del Conocimiento (RUSC), 4(2), 1-11. Recuperado de http://www.uoc.edu/rusc/4/2/dt/esp/ velasquez.pdf

Velandia-Mesa, C., Serrano-Pastor, F., y Martínez-Segura, M. (2017). La investigación formativa en ambientes ubicuos y virtuales en Educación Superior. Comunicar, 51, 09-18. doi: https://doi. org/10.3916/C51-2017-01

Veletsianos, G., y Shepherdson, P. (2016). A Systematic Analysis and Synthesis of the Empirical MOOC Literature Published in 2013-2015. The International Review of Research in Open and Distributed Learning, 17(2), 197-221. doi: 10.19173/ irrodl.v17i2.2448.

Vera Muñoz, M. A. M. (2014). Redes de conocimiento un apoyo para grupos de investigación. Revista SEECI 2OOO, № Extra, 9-17. doi: http://dx.doi. org/10.15198/seeci.2014.35E.9-17

Vuopala, E., Hyvönen, P., y Järvelä, S. (2016). Interaction forms in successful collaborative learning in virtual learning environments. Active Learning in Higher Education, 17(1), 25-38.

Zonoubi, R., Eslami Rasekh, A., y Tavakoli, M. (2017). EFL teacher self-efficacy development in professional learning communities. System 66, 1-12. doi: 10.1016/j.system.2017.03.003. 


\section{PERFIL ACADÉMICO Y PROFESIONAL DE LOS AUTORES}

Ana García-Valcárcel. Catedrática de Tecnología Educativa de la Universidad de Salamanca, en el área de Didáctica y Organización Escolar, es directora del Máster Las TIC en Educación: análisis y diseño de procesos, recursos y prácticas formativas. Dirige el grupo de Investigación e Innovación en Tecnología Educativa de la Universidad de Salamanca (GITE-USAL). Líneas de trabajo: procesos de integración de las tecnologías digitales en la educación y formación del profesorado en competencias digitales.

E-mail: anagv@usal.es

\section{DIRECCIÓN DE LA AUTORA}

Facultad de Educación

Paseo de Canalejas, 169

37008 Salamanca (España)

Luis María González Rodero. Profesor Colaborador de Tecnología Educativa y Organización del Centro Escolar de la Universidad de Salamanca, en el área de Didáctica y Organización Escolar. Es Secretario de la Escuela de Magisterio de Zamora. Miembro del grupo de Investigación e Innovación en Tecnología Educativa de la Universidad de Salamanca (GITE-USAL) desde sus inicios. Líneas de trabajo: procesos de integración de las tecnologías digitales en la educación, didáctica de la formación online y desarrollo de nuevas metodologías de enseñanza-aprendizaje mediadas con TIC.

E-mail: grodero@usal.es

\section{DIRECCIÓN DEL AUTOR}

Escuela de Magisterio de Zamora

Avda. Cardenal Cisneros, 34

49029 Zamora (España)

Verónica Basilotta Gómez-Pablos. Personal Investigador en Formación en la Facultad de Educación de la Universidad de Salamanca, en el área de Didáctica y Organización Escolar, con una ayuda predoctoral de la Junta de Castilla y León, cofinanciada por el Fondo Social Europeo. Miembro del Grupo de Investigación GITE-USAL. Líneas de trabajo: el aprendizaje basado en proyectos en la escuela, el aprendizaje colaborativo y la integración de las tecnologías en los procesos de aprendizaje.

E-mail: veronicabgp@usal.es 
Marta Martín del Pozo. Personal Investigador en Formación con una ayuda predoctoral FPU del Ministerio de Educación, Cultura y Deporte, en el Departamento de Didáctica, Organización y Métodos de Investigación de la Universidad de Salamanca. Miembro del Grupo de Investigación GITE-USAL. Licenciada en Pedagogía y Máster TIC en Educación por la Universidad de Salamanca. Realizando tesis doctoral sobre videojuegos, aprendizaje colaborativo y formación docente. Líneas de investigación son: videojuegos como herramienta de aprendizaje, uso de las TIC en educación y formación docente.

E-mail: mmdp@usal.es

\section{DIRECCIÓN DE LAS AUTORAS}

Facultad de Educación

Paseo de Canalejas, 169

37008 Salamanca (España)

Fecha de recepción del artículo: 02/12/2017

Fecha de aceptación del artículo: 19/01/2018

\section{Como citar este artículo:}

García-Valcárcel Muñoz-Repiso, A., González Rodero, L. M., Basilotta GómezPablos, V., Martín del Pozo, M. (2018). REUNI+D: una red universitaria para la construcción colaborativa de conocimiento. RIED. Revista Iberoamericana de Educación a Distancia, 21(2), pp. 159-177. doi: http://dx.doi.org/10.5944 ried.21.2.20605 